Urszula Kinga Zawadzka

\title{
LA RÉFORME DES FINANCES PUBLIQUES EN FRANCE
}

\section{L'introduction}

Dans la plupart des pays du globe les systèmes financiers publics font l'objet de réformes plus ou moins importantes. En France, on a tenté de transformer le système budgétaire depuis les années soixante, sans presque aucun résultat. L'ordonnance du 2 janvier $1959^{1}$ portant loi organique relative aux lois de finances a servi de la constitution financière pendant presque cinquante ans. La loi organique relative aux lois des finances du $1^{\text {er }}$ août $2001^{2}$ a remplacé l'ordonnance et a provoqué une « révolution budgétaire ». La LOLF est tellement importante parce qu'elle définit un nouveau cadre budgétaire et introduit la réforme de la gestion publique à la fois. Après la présentation des étapes de la réforme seront analysés ses causes et ses principes directeurs.

\section{Les étapes de la réforme}

Pendant près d'un demi-siècle les règles des finances publiques en France ont été déterminées par l'ordonnance du 2 janvier 1959 portant loi organique relative aux lois de finances. Dans ce temps, presque quarante initiatives de la réforme émanant des parlementaires ont échoué. Enfin, le 11 juillet 2000, M. Didier Migaud, député socialiste, rapporteur général de la commission des Finances, a déposé à l'Assemblée nationale la proposition de la loi organique $\mathrm{n}^{\circ} 2540$ relative aux lois de finances (LOLF). Il est important de souligner que le projet de cette loi n'était pas rédigé par le gouvernement. «Le fait que la LOLF soit d'initiative parlementaire lui confère une double force : celle d'éviter la remise en cause lors du changement de la majorité gouvernementale et celle d'impliquer le Parlement dans la réforme afin

1 L'ordonnance $n^{\circ}$ 59-2 du 2 janvier 1959 portant loi organique relative aux lois de finances (JORF du 3 janvier 1959, page 180)

2 La loi organique $n^{\circ}$ 2001-692 relative aux lois de finances (JORF $n^{\circ} 177$ du 2 août 2001, p 12480, texte $n^{\circ} 1$ ). 
d'éviter que les administrations, laissées à elles-mêmes, ne neutralisent la réforme comme elle l'ont fait de presque toutes les tentatives précédentes. $\rangle^{3}$

Le projet de la loi a été discuté à l'Assemble nationale et au Sénat entre février et juin 2001. La LOLF a été adoptée en juin par une majorité parlementaire de gauche en accord avec l'opposition de droite. Le 25 juillet 2001, le Conseil constitutionnel a reconnu la conformité de l'essentiel des dispositions de cette loi, par la décision $\mathrm{n}^{\circ}$ 2001-448 DC. Le $1^{\text {er }}$ août 2001 «la nouvelle constitution financière » a été promulguée.

Les dispositions de la LOLF sont entrées en vigueur en plusieurs étapes. Dix-

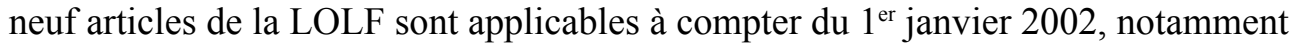
les dispositions relatives au principe de sincérité, aux rapports joints au projet de la loi de finances et au projet de la loi de finances rectificative, aux dispositions élargissant les pouvoirs de contrôle des commissions des finances, à la procédure d'examen du projet de la loi de finances (PLF) et du projet de la loi de règlement. Au $1^{\text {er }}$ janvier 2003 entrent en vigueur les dispositions relatives au débat parlementaire (le débat d'orientation budgétaire) pendant lequel est discuté un rapport sur l'évolution de l'économie nationale et sur les orientations des finances publiques. Depuis le $1^{\text {er }}$ janvier 2004 sont applicables les dispositions sur l'obligation de déposer toutes les disponibilités des collectivités territoriales et leurs établissements publics auprès de l'État. Le projet de la loi de finances pour 2005 était voté en double présentation : selon l'ordonnance de 1959 et selon les principes posés par la LOLF. L'ensemble de la loi organique relative aux lois de finances est en vigueur à compter du $1^{\text {er }}$ janvier 2005. Le projet de la loi de finances pour 2006 était donc le premier entièrement conçu, élaboré et adopté selon les nouvelles règles de la LOLF.

\section{Les causes de la réforme}

L'obsolescence du texte de l'ordonnance portant loi organique relative aux lois de finances du 2 janvier 1959 était une des causes majeures de la nécessité de réformer le système des finances publiques en France. «Prise dans le cadre de la fin des années 1950, dominée par une conception très centralisatrice et très interventionniste de l'Etat, l'ordonnance en porte inconsciemment les marques, reflétant en effet les préoccupations et les illusions de la société d'après guerre, alors qu'elle a continué à s'appliquer à un environnement dans lequel des valeurs souvent inverses avait pris le pas $»^{4}$. Les changements juridiques, financiers et économiques qui avaient lieu en France ont provoqué l'inadéquation du système budgétaire

\footnotetext{
3 C. Rochet, Une seule flèche pour deux cibles: le pari ambitieux de la réforme budgétaire en France, «Management International », n 9, 2004, p. 86.

4 M. Bouvier, M.-Ch. Esclassan, J.-P. Lassale, Finances publiques, Paris, 2002, p. 27.
} 
à la réalité. L'architecture budgétaire reflétant le découpage par ministères et les structures administratives privilégiait les moyens au détriment des objectifs et des résultats. De plus, il est important de mentionner « l'écart toujours croissant entre un système budgétaire étatique juridico-technique, s'appliquant à lui-même des règles autonomes, et un environnement économique et international qui obéit davantage à une logique de gestion. ${ }^{5}$ » Le droit français devrait être donc ajusté surtout aux régulations de l'Union européenne.

Parmi d'autres prémisses de la réforme se trouvent la volonté du pouvoir législatif de renforcer ses compétences en matière de l'élaboration et du contrôle de l'exécution du budget. Depuis longtemps, les autorisations parlementaires ont été privées de leur signification et une situation budgétaire peu transparente a privilégié le point de vue du ministère des Finances. En outre, les informations transmises au Parlement ne lui permettaient pas d'effectuer le contrôle effectif de l'utilisation des deniers public.

Enfin, comme l'a expliqué dans l'exposé des motifs de la proposition de la LOLF M. Didier Migaud, l'ordonnance du 1959 n'a pris guère en compte le fait que l'Etat joue un rôle important dans le financement et l'encadrement de la sécurité sociale qui, depuis la loi constitutionnelle du 22 février 1996, est réglée par une loi spécifique de financement. Or, aujourd'hui, tous les observateurs, notamment à l'échelon de l'Union européenne, s'intéressent à la notion d'" administrations publiques », qui suppose une appréhension globale des opérations financières de l'Etat mais aussi de la sécurité sociale et des collectivités territoriales. ${ }^{6}$

\section{Les principes directeurs de la réforme}

La LOLF a reformé à la fois le système budgétaire et la gestion publique. La réforme budgétaire a visé à rendre possible au Parlement de mieux exercer ses pouvoirs qui sont devenues fantomatiques. Il n'était pas nécessaire d'introduire une nouvelle répartition des rôles entre le Gouvernement et le Parlement car ceux-ci étaient bien marqués. ${ }^{7}$ Les mesures facilitant l'exercice des pouvoirs d'autorisation et de contrôle consistent en :

a) L'introduction du principe de la justification au premier euro. Grâce à la distinction entre les mesures nouvelles et « les services votés » (auparavant 95\% des crédits en moyenne), le Parlement peut se prononcer sur toutes

$5 \quad$ M. Bouvier, M.-Ch. Esclassan, J.-P. Lassale, Finances publiques, Paris, 2002, p. 232.

6 Proposition de la loi organique relative aux lois de finances présentée le 11 juillet 2000 par M. Didier Migaud, Document de l'Assemblée nationale mis en distribution le 12 juillet 2000, $n^{\circ} 2540$.

7 A. Lambert, Une première expérience riche d'enseignement, « Revue Française de Finances Publiques », $n^{\circ} 94$, 2006, p. 35. 
les dépenses au premier euro. «Les services votés » faisant objet d'un seul vote ont induit la reconduite automatique des budgets de fonctionnement ${ }^{8}$. Seulement les nouvelles mesures ont pu être réduites où rejetées par le Parlement.

b) L'élargissement du droit d'amendement parlementaire. A présent, selon l'article 47 de la LOLF, l'irrecevabilité financière résultant de l'article 40 de la Constitution 9 s'apprécie par rapport à la mission. Les parlementaires peuvent redéployer des crédits entre les programmes au sein d'une même mission sous réserve de ne pas majorer le plafond des dépenses de la mission. «Les parlementaires ont fait un usage modéré mais significatif de cette nouvelle liberté ; comme le relèvent tant les rapports de la MILOLF (juin 2006) pour l'Assemblée nationale que celui de la Commission des Finances pour le Sénat (avril 2006) ». ${ }^{10}$

c) Le caractère contradictoire du débat budgétaire qui n'est plus la suite des monologues mais une véritable discussion par mission ministérielle ou interministérielle.

d) La remise en relations des questions financières, qui n'intéressent guère les parlementaires, avec les politiques publiques financées, qui les intéressent bien davantage, alors que les deux sujets paraissaient déconnectés ${ }^{11}$. LOLF « concerne toutes les commissions parlementaires, non les seules commissions des finances. Il n'y a plus, désormais, d'un coté la commission des finances qui se concentre sur les crédits et, de l'autre, les commissions « pour avis » qui ne considèrent que le «fond» de la politique sectorielle suivie. Toutes les commissions doivent désormais apprécier l'adéquation des résultats aux moyens $\rangle^{12}$.

e) L'information budgétaire qui est enrichie. Le Parlement est informé sur tous les mouvements de crédits en exécution, il émet un avis sur les projets de décret d'avance, la procédure des questionnaires budgétaires plus formalisée, au projet de loi de finances de l'année sont joints un rapport sur la situation

8 C. Rochet, Une seule flèche pour deux cibles: le pari ambitieux de la réforme budgétaire en France, « Management International », n 9, 2004, p. 86.

$9 \quad$ Art 40 de la Constitution : "Les propositions et amendements formulés par les membres du Parlement ne sont pas recevables lorsque leur adoption aurait pour conséquence soit une diminution des ressources publiques, soit la création ou l'aggravation d'une charge publique. ».

$10 \mathrm{H}$. Bied-Charreton, X. Hurstler, C. Wendling, S.Magne, Un premier bilan de la préparation et de l'exécution de la loi de finances sous le régime de la loi organique relative aux lois de finances du 1er août 2001, "Revue Française de Finances Publiques », n 96, 2006, p. 186.

11 A. Lambert, Une première expérience riche d'enseignement, «Revue Française de Finances Publiques », n 94 , 2006, p. 33-34.

12 J. Arthuis, La première discussion budgétaire en « mode LOLF » : un pouvoir d'arbitrage exercé par le Parlement, «Revue Française de Finances Publiques », n 94, 2006, p. 20. 
et les perspectives économiques, sociales et financières de la nation et autres annexes.

f) Le principe de sincérité budgétaire (art. 27 et 32 de la LOLF) qui est « moins une innovation qu'une codification de la jurisprudence antérieure du Conseil constitutionnel $\left.{ }^{13}{ }^{1}\right\rangle$

g) La reddition des comptes et évaluation des résultats avant le vote du budget suivant.

Par contre, la voie de l'amélioration de la gestion publique a conduit par le passage de 1'Etat d'une logique de moyens à une logique de résultats. Notamment, il était donc nécessaire de modifier radicalement le principe de spécialité en instaurant de véritables programmes ministériels pour que l'utilisation des deniers publics soit plus efficace. Les crédits ne sont plus divisés par les ministères et les structures administratives (présentation par chapitres). Sous le régime lolfien la nomenclature budgétaire se fonde sur le regroupement des politiques publiques par finalités, le budget est donc structuré en missions, programmes et actions. Une mission, qui est l'unité de vote au Parlement, regroupe un ensemble de programmes et d'actions concourant à une politique publique définie. Un programme, étant l'unité de l'amendement pour les parlementaires, regroupe les crédits destinés à mettre en oeuvre une action ou un ensemble cohérent d'actions relevant d'un même ministère. A chaque programme sont associés une stratégie, des objectifs précis et des indicateurs de performance, placés dans les projets annuels de performance (PAP) grâce à qui est mesurée la réalisation des actions (la performance). Ils sont annexés au projet de la loi de finances initiale. Pour mesurer les écarts entre les prévisions et l'exécution sont préparés les rapports annuels de performance (RAP), annexés au projet de la loi de règlement.

La LOLF organise le budget en programme sans se référer toujours aux institutions administratives. C'est pourquoi il était nécessaire de créer une nouvelle fonction publique de responsables des programmes. Leur tâche consiste à allouer les crédits entre les gestionnaires des programmes (les responsables des budgets opérationnels de programmes) en fonction des objectifs poursuivis et rendre compte des résultats. ${ }^{14}$ Les responsables de programme sont à la tête de masses financières importantes, ce qui leur confère une grande latitude d'actions et permet de s'affranchir des pesanteurs décisionnelles pour gagner en initiatives stratégiques ${ }^{15}$. Ils sont libres de redéployer les crédit appartenant à leur programme entre leurs actions en

13 M. Bouvier, M.-Ch. Esclassan, J.-P. Lassale, Finances publiques, Paris, 2002, p. 275.

$14 \mathrm{H}$. Bied-Charreton, X. Hurstler, C. Wendling, S.Magne, Un premier bilan de la préparation et de l'exécution de la loi de finances sous le régime de la loi organique relative aux lois de finances du 1er août 2001, "Revue Française de Finances Publiques », n 96, 2006, p.187.

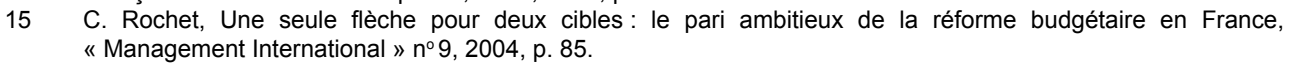


respectant le principe de la « fongibilité asymétrique » qui suppose que l'affectation de certains crédits n'est pas prédestinée de manière rigide, mais prévisionnelle. Les crédits de personnels peuvent être transformés en crédits de fonctionnement, mais non l'inverse. Au sein d'un programme seulement les crédits de personnels ont donc le caractère limitatif.

De plus, la réforme était centrée sur la responsabilisation des gestionnaires et sur le contrôle de leur performance. La responsabilisation des gestionnaires publics, qui est un des principes de base de la LOLF, est constituée de deux volets. Le premier consiste à donner aux gestionnaires plus de liberté, « la responsabilisation s'accompagne ainsi progressivement d'un allègement des procédures, de la réduction des contrôles a priori et de la simplification de la réglementation, en vue d'une plus grande efficacité de l'action administrative et d'une meilleure performance des services public $\gg{ }^{16}$ Selon le deuxième volet, les gestionnaires publics doivent être responsables des résultats qu'ils obtiennent et dont ils sont obligés de rendre compte.

\section{Les conclusions}

Avec la LOLF, on a mis en place en France une nouvelle gouvernance financière publique. Ce n'est pas seulement un texte technique définissant les cadres dans lequel sont élaborées, adoptées et exécutées les lois de finances. La «nouvelle constitution financière» a introduit la réforme centrée sur une grande liberté et une forte responsabilisation des gestionnaires, et le contrôle de leur performance.

De plus, la LOLF a amélioré l'information budgétaire destinée aux citoyens et à leurs représentants et permet d'obtenir des informations difficiles à recueillir auparavant telles que les moyens financiers et humains affectés par l'Etat aux politiques, ce qui rend les finalités des actions publiques plus lisibles. Cependant, dans un rapport paru en mars 2005, les sénateurs soulignent un problème non résolu : « la LOLF n'adopte pas l'approche globale de l'action publique. Or, certaines missions publiques sont partagées entre plusieurs acteurs : Etat, collectivités territoriales, organismes de sécurité sociale. C'est le cas notamment des domaines sanitaire et social, des transports ou encore de la formation professionnelle. A ce jour, il n'est pas possible d'apprécier l'impact global des dépenses des autres organismes publics et donc d'une politique publique dans son ensemble $» .{ }^{17}$

\footnotetext{
16 A. Froment-Meurice, N. Groper La responsabilité des acteurs de la gestion publique en matière budgétaire, financière et comptable : I'heure du bilan, «Actualité juridique - droit administratif », 2005, p. 714.

17 J. Arthuis, La LOLF : culte des indicateurs ou culte de la performance?, rapport d'information no 220 (2004-2005) déposé le 2 mars 2005.
} 
Enfin, dans l'exposé des motifs de la proposition de la loi organique visant à permettre le retour à l'équilibre budgétaire présentée le 15 juillet 2008 le sénateur Bruno Retailleau décrit la situation dangereuse : «Depuis la fin des années 70, la France a accumulé une dette considérable : près de 1200 milliards d'euros. Durant cette période, celle-ci a été multipliée par cinq et a atteint près de la $2 / 3$ du PIB français ${ }^{18} . \gg$ Certains auteurs convainquent que la LOLF à plus long terme offre des instruments en vue de la réduction des déficits publics ${ }^{19}$. Il est donc toujours nécessaire de suivre de manière conséquente les reformes commencées par la LOLF et de chercher les méthodes plus efficaces de gérer les ressources et les dépenses publiques pour que la situation financière de la France soit améliorée.

18 La proposition de la loi organique visant à permettre le retour à l'équilibre budgétaire présenté par M. Bruno Retailleau du 15 juillet 2008, n 467 (2007-2008), site Internet http://www.senat.fr/leg/ppl07-467.html du 22 juin 2008.

19 P. Marini, La loi organique relative aux lois de finances (Lolf), un outil pour la réforme de l'État, « Sociétal » no 53 , 2006, p. 34. 


\section{Streszczenie}

Próby reformowania systemu finansowego we Francji były podejmowane od wczesnych lat 60 . Przez pięćdziesiąt lat finanse publiczne były regulowane przez ordonans nr 59-2 z 2 stycznia 1959 r. ustanawiający konstytucyjną ustawę o ustawach budżetowych. Nieadekwatność tego aktu do zmian następujących na różnych płaszczyznach (prawnej, finansowej i gospodarczej) dawały asumpt do podejmowania prac nad nową ustawą. Po wielu próbach, 1 sierpnia 2001 r. została uchwalona nowa ustawa (loi organique relative aux lois des finances, w skrócie LOLF). Jej postanowienia stopniowo wchodziły w życie w pięciu etapach (pomiędzy 2001 a 2005 rokiem). Ustawa budżetowa na 2006 tok była pierwszą w pełni uchwaloną na postawie LOLF, która zreformowała zarówno system budżetowy, jak i zarządzanie publiczne. 